\title{
Deformation Behavior of a Polygonal Tube under Oblique Impact Loading
}

\author{
Yohei SHINSHII,a, Makoto MIYAZAKI ${ }^{1, b^{*}}$ and Keisuke YOKOYA ${ }^{2, c}$ \\ ${ }^{1}$ National Institute of Technology, Nagano College, 716 Tokuma, Nagano-city, \\ Nagano 381-8550, Japan \\ 2 Tokyo Institute of Technology, 2-12-1 Ookayama, Meguro-ku, Tokyo 152-8550, Japan \\ asyourunner1920@gmail.com, ${ }^{b}$ miyazaki@nagano-nct.ac.jp, 'ppowerto84@gmail.com
}

\section{Keywords: Dynamic Deformation, Impact Load, Plastic Buckling, Numerical Analysis}

\begin{abstract}
Aluminum tubes are energy-efficient absorbing components and are widely used for framework and reinforcement materials of structures. The effects of the axial length and crosssectional shape on the deformation behavior were investigated. Regarding the axial length, it has changed only to a certain length, and there are few studies on it. This paper deals with the influence of axial length. Also, when an impact is actually applied to the square tube, the impact in the oblique direction must also be taken into consideration. Therefore, the deformation behavior was analyzed by applying impact to the square tube from various angles other than the axial direction. An analysis of the dynamic deformation process of the polygonal tube was made using a finite element method. The results show that the load reached the peak immediately after the weight hit the square tube, then declined gently. The same tendency was obtained even if the axial length was changed. However, as the axial length became longer, the displacement taken to reach the peak load increased. As for the impact in the oblique direction, the peak load was small as compared with the axial direction. The deformation of square tube did not buckle in whole but only partially at any length.
\end{abstract}

\section{Introduction}

Square tubes have been used for framework and reinforcement members of structures. There are many studies on circular tubes, and deformation behaviors have been studied by static and dynamic compression tests [1]. Previous studies have shown that square tubes have a role of absorbing impact energy by crushing under pressure in the axial direction at the time of a collision [2]. Aluminum alloy has a Young's modulus that is one-third that of commonly used steel materials, giving it the disadvantage of low rigidity. In addition, the whole buckles become large when thickness is increased, and causing axial compression deformation, which cannot effectively absorb collision energy[3]. The tubular bodies with polygonal tubes and cellular cross sections have been studied as a means to effectively absorb energy [4]. Additionally, an influence of axial length on dynamic axially compressed aluminum tubes is being considered [57].It is known that elastic deformation occurs in the entire square tube prior to plastic deformation when the square tube deforms. Since this is periodic and wavy, it seems that the axial length will have a large influence. In a previous study, deformation behaviors up to 500 $\mathrm{mm}$ in length have been considered [8]. The purpose of this paper is to discuss, the deformation behavior of dynamic axial compression of an aluminum square tube of axial lengths of $500 \mathrm{~mm}$, $750 \mathrm{~mm}$ and $1000 \mathrm{~mm}$. Also, when an impact is applied to the tube, the impact in the oblique direction must also be taken into consideration. Therefore, for comparison with the axial compression, deformation behavior of aluminum square tube under oblique impact loading was considered. 


\section{Numerical Analysis}

Analytical method. The analysis is conducted by non-linear structure analysis program (Marc 2018) and pre-post processor (Mentat 2018). An example of analytical model is shown in Fig. 1. The specimen is an aluminum tube (A6063-T5). Material properties are shown in Table 1. Concerning axial length $l$, the square tubes $(l=500 \mathrm{~mm}, 750 \mathrm{~mm}$ and $1000 \mathrm{~mm})$ are discretized at 20000, 30000, 40000 bilinear four-node shell elements, respectively. For the analysis in the oblique direction, an angle of $\theta=10$ degrees was given between the weight and the impact edge of the square tube. Schematic diagram of the analysis model is shown in Fig. 2.

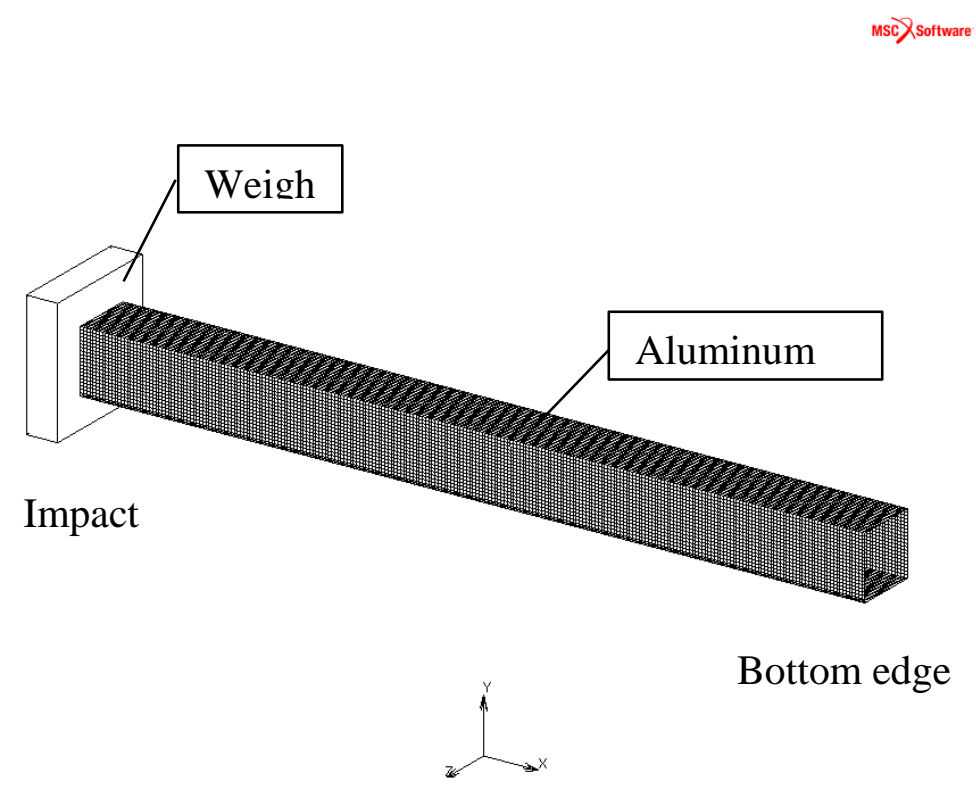

Fig. 1 Analytical model of square tube $(l=500 \mathrm{~mm})$.

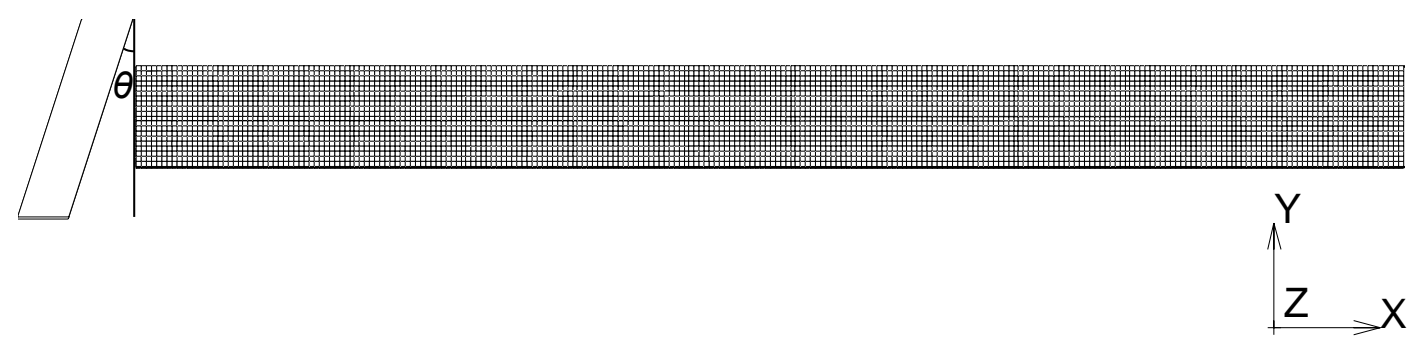

Fig. 2 Impact angle of weight $(\theta=10 \mathrm{deg})$.

The nodes on the edge of the tube are fixed with the exception of in the axial direction of the impact edge. The weight $(80 \times 80 \times 20 \mathrm{~mm}, 15 \mathrm{~kg})$ is an un-discretized three-dimensional, eightnode, first-order, isoparametric element. The deformed tube is regarded as an isotropic material following von-Mises yield condition, and the flow stress-strain relationship is shown in Equation (1) because the effect of the strain rate of the aluminum is smaller than that of other materials like iron, etc [9]. 
Table1 Material properties of aluminum (A6063-T5)

\begin{tabular}{llc}
\hline Young's modulus & $E[\mathrm{GPa}]$ & 69 \\
Poisson's ratio & $v$ & 0.33 \\
Density & $\rho\left[\mathrm{kg} / \mathrm{m}^{3}\right]$ & $2.71 \times 10^{3}$ \\
Work-hardening modulus & $F[\mathrm{MPa}]$ & 268 \\
Work-hardening exponent & $n$ & 0.065 \\
\hline
\end{tabular}

$\sigma=F \varepsilon^{n}$.

In this analysis, the time step width is $1 \mu \mathrm{s}$. The Newton-Raphson method and the updated Lagrangian formulation are used as the solution methods of the non-linear equation, and the single-step houbolt of implicit solution time-integration method is used for the analysis of dynamic deformation. The impact velocity is $10 \mathrm{~m} / \mathrm{s}$.

\section{Results and Considerations}

Analytical results. Final deformations of the square tube $(\mathrm{l}=500 \mathrm{~mm}, 750 \mathrm{~mm}$ and 1000 mm)are shown in Fig. 3.

(a)
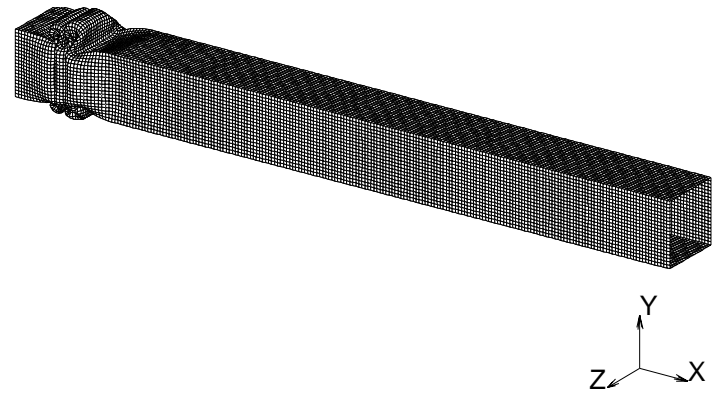

(c)

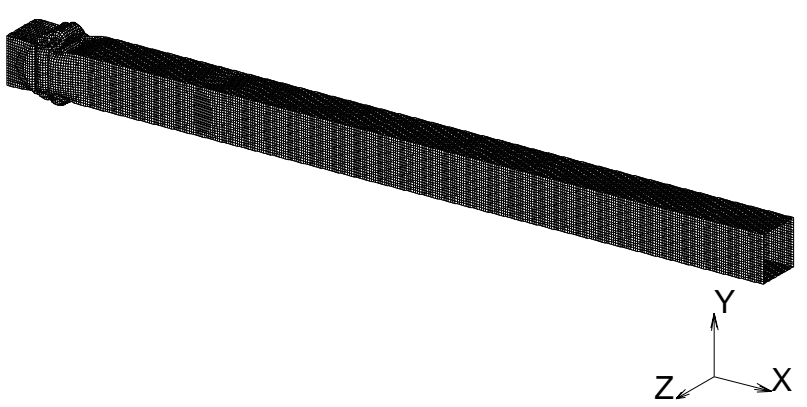

(b)

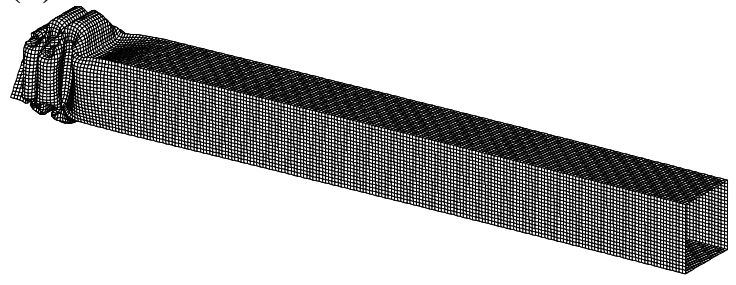

$2 \stackrel{1}{x} x_{x}$

(d)

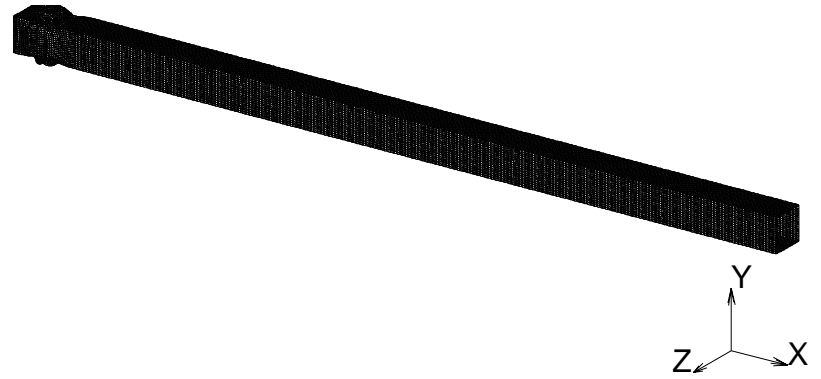

Fig. 3 Final deformations of square tube $(a) l=500 \mathrm{~mm},(b) l=500 \mathrm{~mm}$ (oblique direction), $c) l=$ $750 \mathrm{~mm},(d) \mathrm{l}=1000 \mathrm{~mm}$.

Load-displacement curve. Load-axial displacement curves of square tube with length variation are shown in Fig. 4. The same tendency was obtained even if the axial length was changed. The peak load is not hardly affected by axial length. However, it was confirmed that the displacement taken to reach the peak load is dependent on the axial length. And the final displacement is independent of the axial length. 


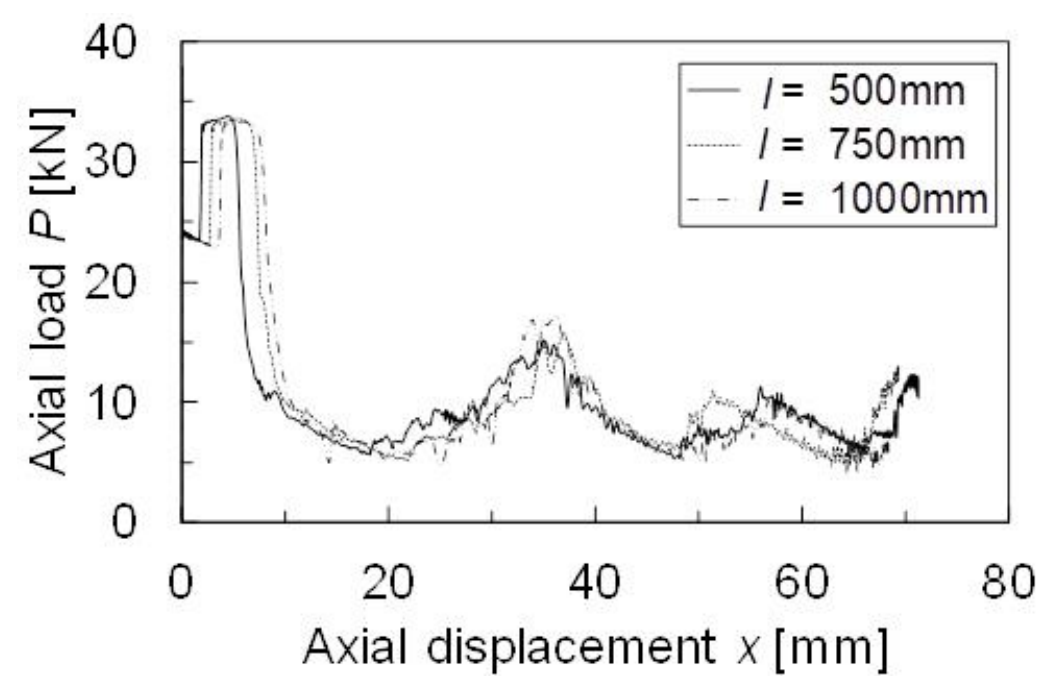

Fig. 4 Relationship between axial load P and axial displacement $x$ (axial loading,l $=500 \mathrm{~mm}$, $750 \mathrm{~mm}$ and $1000 \mathrm{~mm}$ ).

The load-displacement curves in the axial direction and oblique direction of length $l=500$ $\mathrm{mm}$ are shown in the Fig. 5.Compared with the result of loading in the axial direction, the peak load is small. And the displacement taken to reach the peak load is larger than that in the axial direction.This is because the weight collides with the upper portion of the tube so that the initial collision portion is smaller than the axial direction. After that, the load reached a peak at around $17 \mathrm{~mm}$ in axial displacement, because the entire weight collided with the tube.

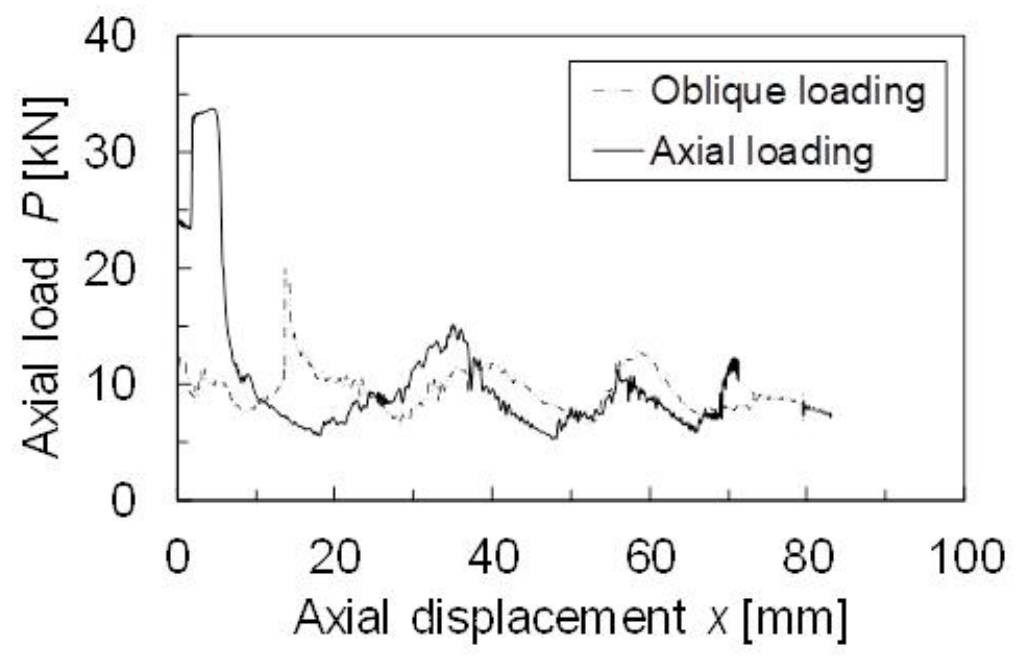

Fig. 5 Relationship between axial load $\mathrm{P}$ and axial displacement $x$ (axial loadingand oblique loading, $\mathrm{l}=500 \mathrm{~mm}$ ).

Effects of axial length on compressive strain distribution. Strain distributions in bucking region are shown in Fig. 6. As seen in Fig. 6, it was confirmed that peak strain wasnot considerably affected by changes in the axial length. The strain occurs partially at the side of the tube even if the axial length changes. In addition, the strain is almost 0 except for the peak strain region. From the above results, it was also confirmed that deformation occurred partially. 


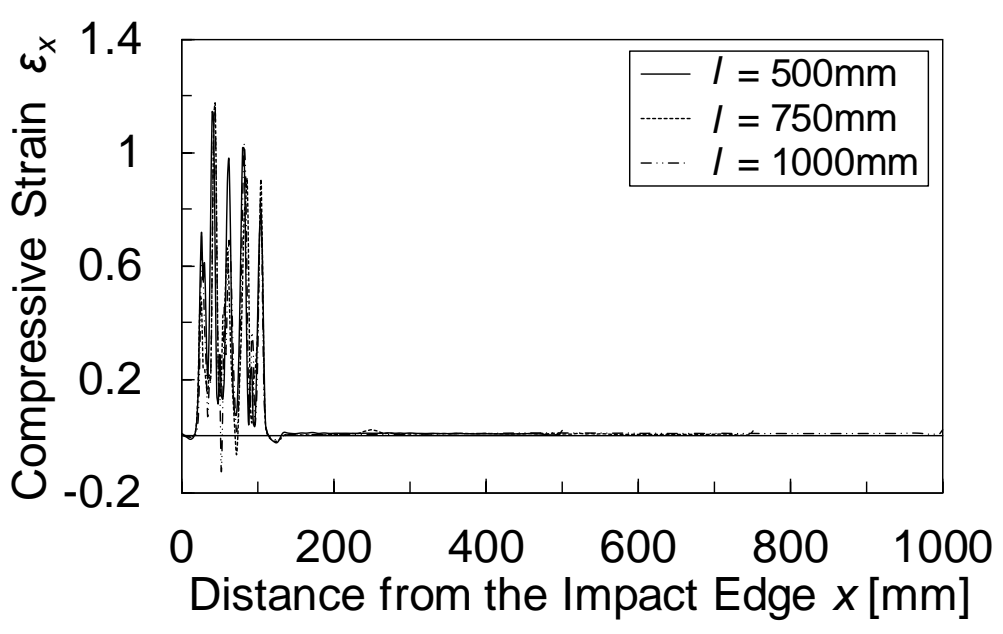

Fig. 6 Compressive strain distribution along the side.

Effects of axial length on axial strain distribution.Strain distributions along the hill and valley are shown in Fig. 7. The distortion here shows the compressive strain at the position where the concave-convex pattern of the edge is maximized.It was confirmed that axial strain near the corner of the tube is large compared to that of the other parts of tube. Even if the axial length changes, the trends of the axial strain distribution was not considerably affected. In addition, the peak strain did not markedly change.

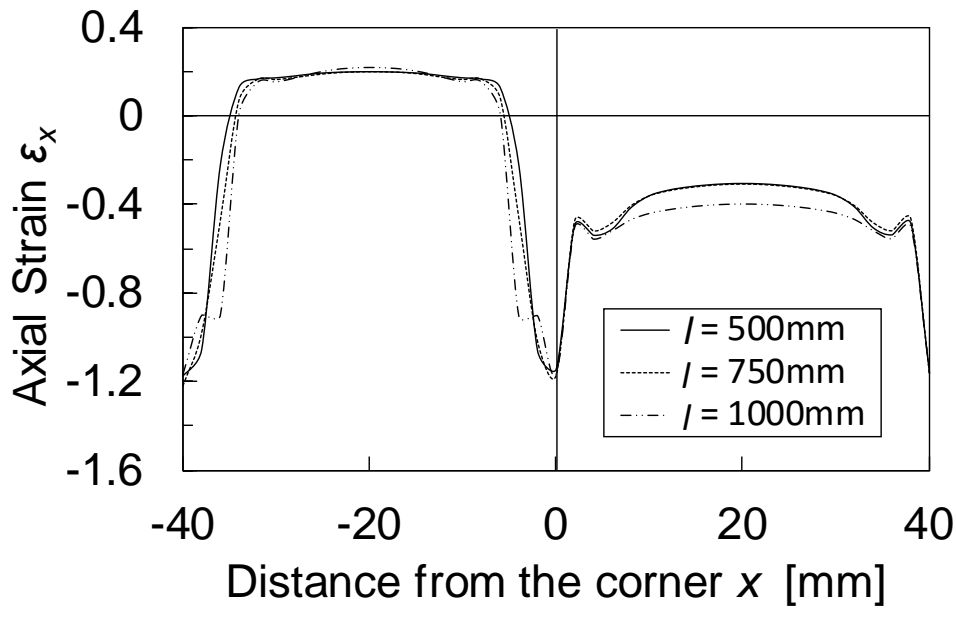

Fig. 7 Axial strain distribution along the hill and valley.

\section{Conclusions}

The deformation behavior of the square tube subjected to dynamic axial compression was compared with the results of examination by finite element analysis with the axial length varying from $500 \mathrm{~mm}$ to $1000 \mathrm{~mm}$ and the deformation behavior in the axial direction and diagonal direction when the axial length was $500 \mathrm{~mm}$. The following conclusions were obtained.

(1) The trends of the load-displacement curve and peak load were not considerably affected by changes in the axial length.

(2) As the axial length increases, the final displacement becomes smaller. 
(3) In the case of oblique direction impact, it was found that the peak load is small compared tothat of axial direction impact, and the displacement taken to reach the peak load is dependent on the axial length.

(4) The deformation of the square tube did not buckle in whole but only partially.

\section{References}

[1] W. Abramowicz and N. Jones, International Journal Impact Engineering. 2-2 (1984) 179-208.

[2] M. Yamashita, H. Kenmotsu and T. Hattori : Thin - Walled Struct., 69 (2013), 45-53.

[3] D-K. Kim, S. Lee and M. Rhee, Materials \& Design. 19-4 (1998) 179-185.

[4] J Fang, Y. Gao, G. Sun, N. Qiu . and Q. Li : Thin-Walled Struct., 95 (2015), 115-126.

[5] M. Miyazaki, H. Endo, and H. Negishi, J. Mater. Process. Technol., 85-1-3 (1999) 213-216.

[6] M. Miyazaki and H. Negishi, Materials Transactions. 44-8 (2003) 1566-1570.

[7] M. Miyazaki and M Yamaguchi, Procedia Engineering. 81 (2014) 1067-1072.

[8] K. Yokoya, M. Miyazaki, Y. Tojo and M. Yamashita : Procedia Eng., 207 (2017), 251-256

[9] S. Tanimura, H. Hayashi, T.Yamamoto and K. Mimura, J. Solid Mech. \& Mater., 3-12 (2009), 1263-1273. 